\title{
Der begleitete Freitod und der Arzt
}

\section{Alois Geiger-Jakob}

Der Autor ist ein für Dignitas rezeptierender Arzt
Korrespondenz:

Dr. med. Alois Geiger-Jakob

Holenbachstrasse 3

CH-8105 Regensdorf

dr.a.geiger@bluewin.ch

\section{Der Suizid als gesellschaftliches Tabu}

Der Suizid wird in unserer westeuropäischen Gesellschaft noch immer als eines der grössten Tabus erlebt, nicht zuletzt auch in der medizinischen Welt. Dies geht wohl auf den über viele hundert Jahre dauernden Einfluss der christlichen Religion zurück, die in dieser Zeit das Moralmonopol innehatte. Bis heute haben wir in deutscher Sprache umgangssprachlich kein neutrales Wort für den Suizid, der die Selbsttötung meint, bei uns aber noch immer Selbstmord genannt wird. Unter Mord verstehen wir jedoch eine besonders verwerfliche Form des Tötens, und so übertragen wir «das Verwerfliche» unbewusst auch auf den Suizid und sogar auf den begleiteten Freitod; einige möchten diesen überhaupt verbieten [1]. In unserer heutigen Gesellschaft ist es aber nicht mehr zulässig, eigene religiöse oder moralische Vorstellungen andern aufzuzwingen, insbesondere dann nicht, wenn jemand deswegen ein Leiden auf sich nehmen soll, von dem er sich befreien will, wohlverstanden zum Preis der Aufgabe seines eigenen Lebens. Für Menschen, die zu einer mehr oder weniger atheistischen Weltanschauung gelangt sind und im Gottesglauben eher einen historisch überlieferten «Gotteswahn» [2] sehen, gibt es nämlich keinen Grund, in einem andauernden und unheilbaren Leiden einen Sinn zu finden, es sei denn als persönliche Herausforderung an ihren eigenen Reifeprozess und an ihre Entwicklung zu einer persönlichen Form der Weisheit hin. Ob eine solche Entwicklung angetreten werden soll oder nicht, muss aber gewiss dem betroffenen Individuum überlassen bleiben. Thomas Morus (der Heilige!) hat diese Möglichkeit in seinem Buch «Utopia» schon im 16. Jahrhundert vorgesehen, indem in seinem Fantasieland jeder Schwerkranke wählen durfte, ob er bis zu seinem Tod mit aller Liebe gepflegt werden oder ob er seinem leidvollen Leben ein selbstbestimmtes Ende setzen wolle. Diese Entscheidung wurde respektiert [3]. Unserem heutigen humanen Denken kommt diese damalige Utopie sehr nahe, und es ist uns selbstverständlich, dass derjenige, der seinem Leben wohlerwogen und nach reiflicher Überlegung ein Ende setzen will, von seinen Mitmenschen im Augenblick seines Sterbens nicht allein gelassen werden soll.

\section{Le suicide accompagné et le médecin}

Le suicide accompagné est affecté par le tabou qui repose sur le suicide depuis des siècles, dû à l'héritage chrétien. Cependant, le suicide accompagné est bien différent du suicide spontané dans l'absence d'autres êtres humains, qui n'est presque jamais discuté d'avance et dont l'acteur reste ainsi isolé et prisonnier du désespoir de la situation momentanée. Le suicide accompagné par contre bénéficie de la nécessité de communication avec ceux qui seront avec le mourant au moment de la fin de sa vie, organisé des semaines et même des mois en avance, soit ses proches et les membres accompagnants de I'organisation choisie. Pour pouvoir mourir en dignité, ces patients ont cependant besoin de l'aide d'un médecin, puisque une mort douce, sereine et sûre n'est possible qu'avec la prescription du médicament nécessaire.

\section{Der begleitete Freitod als Ausdruck des Humanen}

Die Begleitung beim Freitod hat zwei wichtige Aufgaben:

1. Sie entreisst den freiwillig und überlegt vorgenommenen Akt durch die Anwesenheit empathisch gestimmter Mitmenschen der Isolation. Zwischen dem begleiteten Freitod, wie er bei Dignitas oder Exit durchgeführt wird, und dem einsamen, in Verzweiflung durchgeführten Suizid in Abkehr von den Mitmenschen liegen Welten! Einem Sterbewilligen nur die Möglichkeit zum Suizid zu vermitteln genügt nämlich nicht. Nahezu sämtliche Menschen, die einen nichtbegleiteten Suizid in Betracht ziehen, sehen für sich praktisch keine Möglichkeit, sich mit Angehörigen oder Freunden vorgängig über ihre Absicht zu unterhalten (oft nicht einmal mit ihrem Arzt), da der Suizid und bereits die Suizidabsicht 
nach wie vor dem genannten gesellschaftlichen Tabu unterliegen und allenfalls sogar psychiatrische Zwangsmassnahmen nach sich ziehen können. Durch das Fehlen von Mitmenschlichkeit in dieser Situation fehlt ihnen aber ein echter Gedankenaustausch, und die Isolation lässt sie letztlich unfrei handeln. Ganz anders ist die Situation beim begleiteten Freitod, der in grossem Masse durch Zuwendung gekennzeichnet ist.

2. Sie garantiert ein sicheres Sterben. Auch bei gutüberlegten Suiziden in Eigenregie kann Unvorhergesehenes jederzeit den geplanten Ablauf stören, allenfalls mit schweren gesundheitlichen Folgen für die Betroffenen. Dies wird durch die Begleitung vermieden.

\section{Die Rolle des Arztes beim begleiteten Freitod}

Gibt es für den Arzt überhaupt eine Aufgabe beim begleiteten Freitod? Sollen wir Ärzte uns nicht vornehm zurückhalten und die Sterbewilligen ihrem Schicksal überlassen? Dass wir sie am Suizid zumindest nicht mehr hindern sollen, wenn der Wunsch dazu wohlerwogen und konstant ist, ist klar: Das Urteil des Eidgenössischen Bundesgerichts vom 3. November 2006 [4] hält fest, dass das Recht eines Menschen, über Art und Zeitpunkt der Beendigung seines eigenen Lebens zu entscheiden, Bestandteil des Selbstbestimmungsrechts des Menschen ist, das von der Europäischen Menschenrechtskonvention geschützt wird [5]. Es hält allerdings auch fest, dass dieses Recht nicht den Anspruch enthält, das Ausstellen eines Rezeptes zum Beispiel für Natriumpentobarbital $(\mathrm{NaP})$ von einem Arzt fordern $\mathrm{zu}$ dürfen. Gerade durch dieses Diktum sind wir Ärzte jedoch erst recht aufgerufen, uns mit Überlegungen zur entsprechenden Rezeptur zu befassen.

Für einen von Mitmenschen in Nächstenliebe begleiteten Freitod in Würde gibt es nämlich nur wenige ethisch vertretbare Varianten. Alle physikalisch möglichen Varianten sind für die Begleiter mit fast unmenschlich anmutenden und untragbaren Belastungen* verbunden. Die für alle am wenigsten belastende Möglichkeit der Hilfe besteht in der Abgabe eines tödlichen Medikamentes, das den zum Tode Entschlossenen ruhig einschlafen lässt. Dies ist aber an eine Rezeptierung gebunden, so dass wir Ärzte für einen humanen begleiteten Freitod nicht ersetzbar sind. Diese Tatsache nimmt uns in Verantwortung.

\section{Der begleitete Freitod als Prophylaxe verzweifelter Spontansuizide}

Daten zur Suizidfrage in der Schweiz sind rar; bei den Suizidversuchen fehlt es mangels Melde- pflicht an exakten Daten überhaupt. In der Antwort des Bundesrates vom 9. Januar 2002 heisst es, wenn man die Zahlen amtlich registrierter Selbsttötungen mit den geschätzten Dunkelziffern ergänze, «ergeben sich für 1997 rund 20000 bis 67000 versuchte Suizide in der Schweiz» [6]. Auch wenn viele der spontanen Suizidversuche vorwiegend apellativen Charakter haben, bedeutet dies nicht, dass sie in der jeweiligen Situation nicht durchaus ernstgemeint, mit Sicherheit aber oft nicht genügend reflektiert sind. Begleitung beim Suizid setzt Gespräche voraus und damit Reflexion. Wenn der begleitete Freitod möglich ist, hat er daher naturgemäss eine prophylaktische Wirkung gegenüber Suiziden und Suizidversuchen aus Verzweiflung. Ein Mensch, der sich um einen begleiteten Freitod bemüht, hat einen meist langen und den Suizid immer wieder in Frage stellenden Weg vor sich, nicht zuletzt und zumeist auch mit seinen Angehörigen, die beim «gewöhnlichen» Suizid immer von den Tatsachen überrannt werden, beim begleiteten Suizid jedoch am Ende oft doch mit einwilligen können und dann sogar dabei anwesend sind. Welch ein Gewinn für die entsprechende Trauerarbeit dieser Menschen!

\section{Der Ablauf der Freitodbegleitung bei einer Vereinigung wie Dignitas}

Als Arzt, der mit Dignitas zusammenarbeitet, erhalte ich jeweils ein Dossier über ein Mitglied, das seinem Leben ein Ende setzen möchte. Vorher hat es die Mitgliedschaft erworben und mit Dignitas-Mitarbeitern bereits einen intensiven Kontakt erlebt, in dessen Verlauf bereits Alternativen erwogen worden sind. Das Dossier besteht aus einem schriftlichen Gesuch um Vorbereitung des begleiteten Suizids, aussagekräftigen medizinischen Unterlagen sowie einem Lebensbericht. Mir wird die Frage gestellt, ob ich grundsätzlich bereit sei, dem Mitglied unter diesen Umständen ein Rezept zuzusagen.

Meist wird aufgrund der Akten bereits ein recht klares Bild der Erkrankung und der Situation des Patienten möglich, und «grünes Licht» kann gegeben werden, insbesondere wenn klar ist, dass die therapeutischen Möglichkeiten ausgeschöpft sind. Wo jedoch Alternativen bestehen, schlage ich sie vor. Allenfalls lege ich dem Mitglied sogar Bedingungen fest, die zuerst erfüllt werden müssen, bevor ich den Fall erneut beurteile. Nur in sehr seltenen Fällen muss ich die Freitodbegleitung grundsätzlich ablehnen. So erfolgt in diesem zeitlich oft ausgedehnten Vorfeld eine sorgfältige medizinische und allenfalls persönliche Beratung, die gar nicht so selten zu einem Sinneswandel und damit zum Weiterleben führt. 
Trotz Zusage des «grünen Lichts» werden viele Anträge von den Mitgliedern anschliessend nicht mehr weiterverfolgt; ihnen genügt die Zusage, dass eine Freitodbegleitung möglich wäre, um dann doch nicht von ihr Gebrauch zu machen. So bemühen sich nur etwa 30\% der Anfragenden wirklich um einen Termin für die Freitodbegleitung; in nur 13,3\% kommt es dann zur konkreten Planung und später zum Arztgespräch mit dem Mitglied [7].

Das Gespräch umfasst in erster Linie die Beurteilung der Urteilsfähigkeit des Mitglieds in bezug auf seinen Sterbewunsch. Zumeist genügt hierfür ein unstrukturiertes Gespräch, in dem das Mitglied aufgefordert wird, von seiner Situation zu erzählen, zu zeigen, dass es über seine Krankheit und deren Prognose orientiert ist, dass es weiss, wie die Freitodbegleitung ablaufen wird und welch tödliche Konsequenz die Einnahme von Natriumpentobarbital hat. Es wird während des Gesprächs auf die Affektlage und allfälliges Vorhandensein oder eben Nichtvorhandensein von psychopathologischen Symptomen geachtet. Gleichzeitig wird auch der physische Zustand beurteilt und abgewogen, ob dieser zur dokumentierten Krankheit passt. Geachtet wird auch auf Anzeichen einer allfälligen Fremdbestimmung. In Fällen von Unsicherheit zu diesen Punkten können sehr gezielt gestellte Fragen oder Tests nötig werden.

Aufgrund einer Intervention des Zürcher Kantonsarztes braucht es seit einiger Zeit in Zürich nun mindestens ein zweites Gespräch des Arztes mit dem Mitglied. Im Anschluss an die Begegnungen formuliere ich einen Bericht, der über die auswärts gestellten Diagnosen, die Exploration und die erhobenen Befunde Auskunft gibt. Daneben stelle ich das Rezept für 15,0 g NaP (dosis letalis!) zuhanden der Freitodbegleiter aus. Das Rezept wird dem Mitglied nie direkt ausgehändigt; die entsprechenden Akten gehen an die Behörden.

Zum dritten Mal hält das Mitglied für die Durchführung des Suizids nun einen vorher abgemachten Termin ein, so dass alles Unüberlegte und Verzweifelte vom Vorhaben abgefallen ist. Es wird von zwei Freitodbegleitern empfangen, erneut wird miteinander gesprochen, das Mitglied unterzeichnet eine letzte Willenserklärung, erhält eine Prämedikation gegen das Erbrechen. Wann immer der Patient dann zum letzten Schritt bereit ist, kann er das Glas mit in Wasser gelöstem NaP trinken; er kann sich aber auch noch im letzten Moment umbesinnen. Nach dem
Trinken des tödlichen Medikamentes wird er im Bett rasch einschlafen und nicht mehr erwachen. Die Angehörigen sind beim Sterbenden oft mit dabei, halten ihm allenfalls die Hand. Es ist der humanste und sozialste Tod, den man sich vorstellen kann, und wir Ärzte haben die Chance, durch die Verordnung des dafür wirksamsten Mittels dieses sanfte Sterben zu ermöglichen.

\section{Ärztlicher Beistand als Gebot}

Der begleitete Freitod ist für die wenigen Menschen $^{* *}$, die ihn wählen, kein verzweifelter Akt des Sich-Umbringens, sondern ein denkbarer, abgewogener und dann zielstrebig geplanter Entscheid zur Beendigung dieses unerträglich gewordenen Lebens, aufgehoben in der Gemeinschaft von Mitmenschen.

Das Recht auf Suizid ist kein theoretisches Recht, sondern ein sehr konkretes Menschenrecht, und dieses Recht darf deshalb nicht durch zu hohe Hürden bei der Inanspruchnahme oder gar durch Verweigerung der ärztlichen und mitmenschlichen Hilfe illusorisch gemacht werden. Daher soll die Freitodbegleitung auch nicht durch Reglemente oder behördliche Massnahmen weiter erschwert werden. Es bleibt aber eminent wichtig, dass alle Freitodbegleitungen mit ärztlicher Beteiligung vorbereitet werden, weil so das ärztliche Wissen um physische und psychische Krankheiten in die Entscheidung zum Suizid und in dessen Durchführung einfliessen kann. So sind und bleiben wir Ärzte gefordert.

\section{Literatur}

1 Beutler D. Leben im Sterben - eine Stellungnahme zur Suizidbeihilfe. Schweiz Ärztezeitung. 2008; 89(10):411-5.

2 Dawkins R. The God Delusion. London: Bantam Press; 2006

3 Morus T. Utopia. Patron der Regierenden und der Politiker. 1516.

4 BGE 133 I 58.

5 EMRK Artikel 8 Absatz 1.

6 Antwort des Bundesrates auf die Einfache Anfrage Andreas Gross (SP ZH) betr. Suizide und Suizidversuche vom 9. Januar 2002.

7 Diplomarbeit an einer katholischen deutschen Fachhochschule, anonym veröffentlicht von Dignitas am 23. April 2007.

8 Bosshard G, Ulrich E, Bär W. 748 cases of suicide assisted by a Swiss right-to-die organisation. Swiss Med Wkly. 2003;133(8):310-7. 